\title{
April 2015 Critical Care Case of the Month: Half-Sided Light House
}

\author{
Theodore Loftsgard APRN, ACNP, CCRN \\ Adam Frost RRT, CRT \\ Dacia Evans RN \\ Karen Kolbet PharmD, RPh
}

\author{
Division of Critical Care \\ Mayo Clinic \\ Rochester, Minnesota
}

\section{History of Present IIIness}

A 55 year old woman was transferred to the ICU from the general medicine ward for tachycardia and acute hypoxic respiratory distress. She has multiple myeloma and had received cycle one of bortezomib, dexamethasone, thalidomide, cisplatin, doxorubicin, cyclophosphamide and etoposide (VDT-PACE) and radiotherapy to T7 for a pathologic compression. She was admitted for pain control from mucositis.

\section{Past Medical History}

In addition to the multiple myeloma she has a past medical history of asthma, ovarian cysts, diverticulitis, eczema, pneumonia, laparoscopic cholecystectomy, total abdominal hysterectomy with bilateral salpingo-oophorectomy, appendectomy, ectopic pregnancy in the past, and left Bell's palsy.

\section{Current Medications}

- Acyclovir 400 mg BID

- Albuterol 90 HFA prn

- Allopurinol $300 \mathrm{mg}$ daily

- Fluconazole $200 \mathrm{mg}$ BID

- Gabapentin 300 mg BID,

- Hydromorphone

- Levofloxacin 500 daily

- Morphine

- Omeprazole

- Bactrim 400-80 mg daily for PCP prophylaxis

- Thalomid $200 \mathrm{mg}$ capsule daily

- Ativan $0.5 \mathrm{mg}$ just prior to transfer

\section{Physical Examination}

Vital Signs: temperature $36.4{ }^{\circ} \mathrm{C}$, respiratory rate 24 breaths/minute, blood pressure $148 / 77 \mathrm{~mm} \mathrm{Hg}$, pulse $133 /$ minute, $\mathrm{SpO} 298 \%$ on oxygen at $4 \mathrm{~L} / \mathrm{min}$.

General: Alert and follows commands. Slightly somnolent. In respiratory acute distress. Skin: Pink, warm and dry without acute rashes or lesions.

Eyes: EOMs intact. Conjunctivae pink. Sclerae anicteric

ENT: Neck supple. Trachea midline. 
Cardiac: S1, S2 irregular rate and rhythm without extra sounds, murmurs, rubs or gallops. Capillary refill 2 seconds.

Lungs: Respirations with accessory muscle use, shallow. scattered crackles and equal to auscultation. Diminished bilateral bases.

Abdomen: Soft. No abdominal tenderness. Non-distended. Bowel sounds present. Extremities: Peripheral pulses $+2 / 4$ throughout. $1+$ peripheral edema.

Neuro: GCS $=13$, residual bell's palsy.

\section{Pertinent Labs}

Sodium: $144 \mathrm{mmol} / \mathrm{L}$

Potassium: $4.2 \mathrm{mmol} / \mathrm{L}$

Chloride: $113 \mathrm{mmol} / \mathrm{L}$ *

Bicarbonate,: $23 \mathrm{mmol} / \mathrm{L}$

Creatinine: $0.6 \mathrm{mg} / \mathrm{dL}$

Hematocrit: $20.5 \%$

Leukocytes: $0.5 \times 10(9) / L$

Hemoglobin: $6.2 \mathrm{~g} / \mathrm{dL}$

Platelet Count: $39 \times 10(9) / \mathrm{L}$

Calcium, lonized(S): $4.81 \mathrm{mg} / \mathrm{dL}$

pH (FOR CALCIUM, IONIZED [S]): 7.47

INR: 1.5

APTT(P): $29 \mathrm{sec}$

Her ECG (Figure 1) showed a tachycardia with a maximum heart rate was in the 170's.

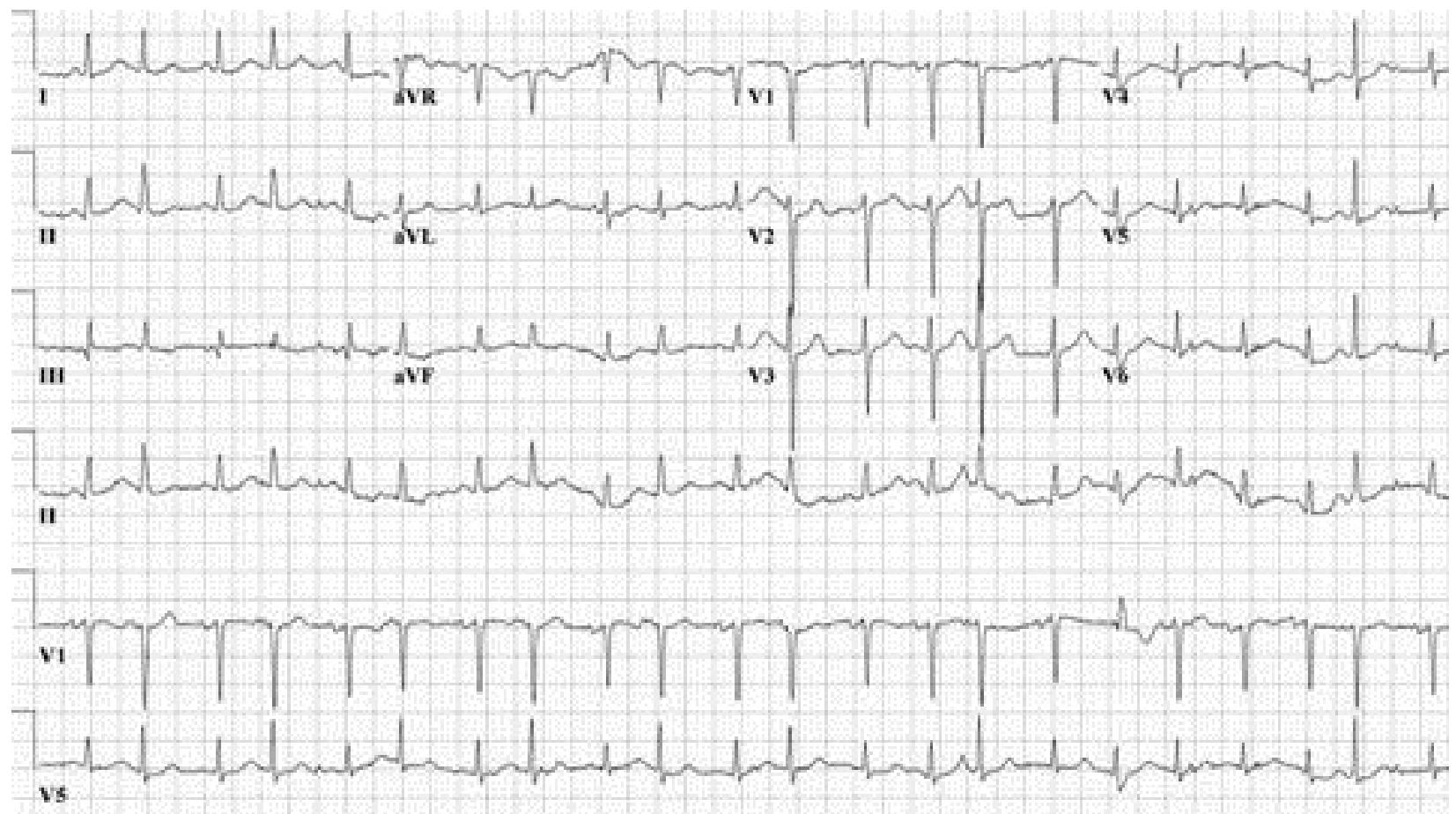

Figure 1. Admission ECG to the ICU. 
Her admission chest x-ray is shown in Figure 2.

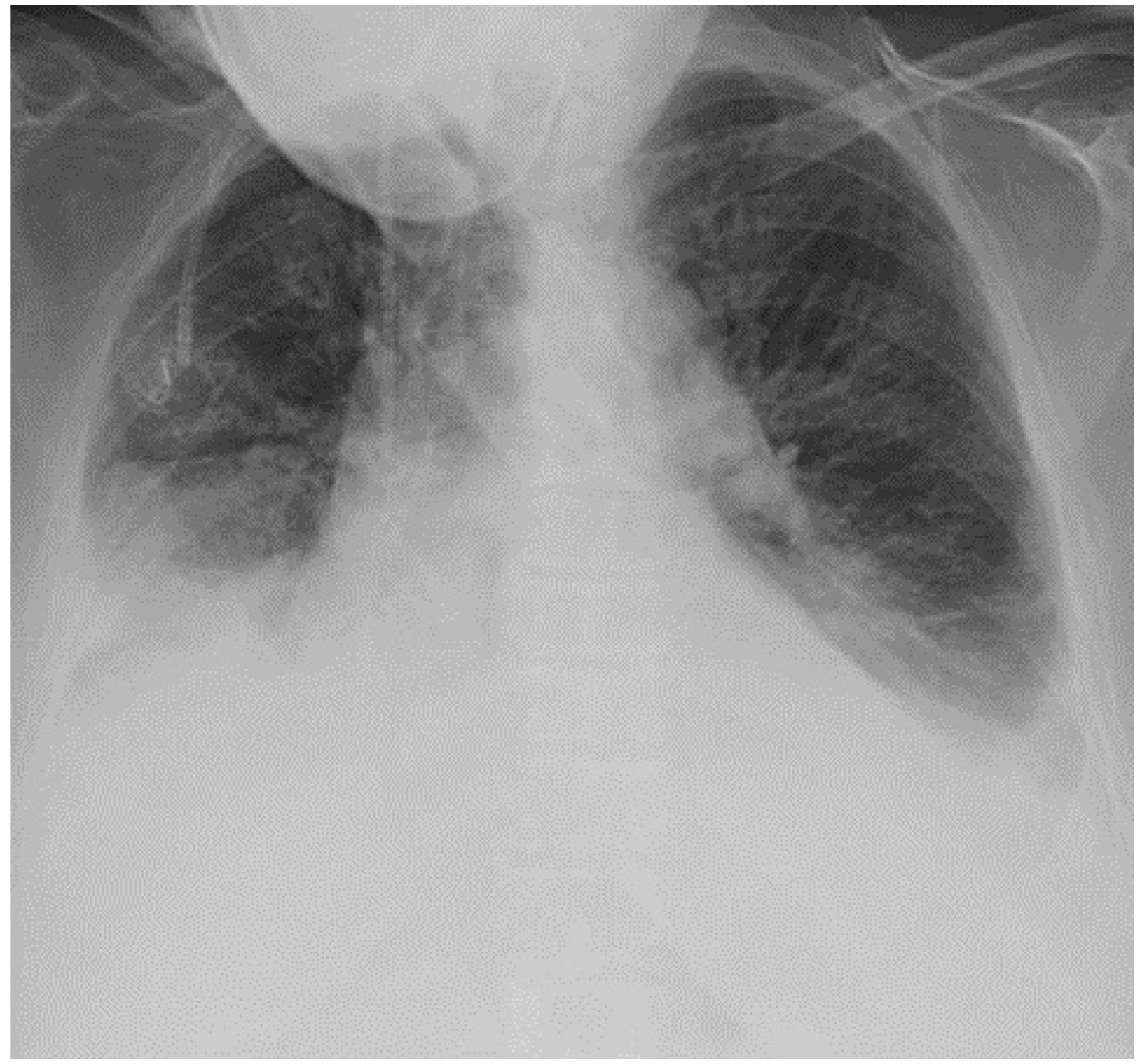

Figure 2. Portable chest x-ray taken just after admission to the ICU.

Which of the following are true?

1. The EKG shows atrial fibrillation with a rapid ventricular response

2. She should be immediately intubated for airway protection

3. The chest $x$-ray shows bilateral pleural effusions

4. 1 and 3

5. All of the above 


\section{Correct!}

\section{1 and 3}

Her ECG shows an irregular rhythm with a rapid ventricular response. There are no consistent $P$ waves proceeding the QRS complex consistent with atrial fibrillation. She was given $10 \mathrm{mg}$ of IV metropolol and her rate decreased to 100-110 and became regular.

Neither diaphragm is identifiable on the portable chest x-ray and there appears to be volume loss with atelectasis. This suggests bilateral pleural effusions.

She is awake, able to follow commands and probably able to protect her airway. CPAP $7 \mathrm{~cm} \mathrm{H} 2 \mathrm{O}, 30 \%$ was applied with some improvement.

She was transfused with 2 units of packed red blood cells and had a right-sided thoracentesis which removed 1 liter of straw-colored fluid. After removal of the fluid she had increasing shortness of breath. A lung ultrasound was performed (Figure 3). Lung sliding was seen on both the left and right side.

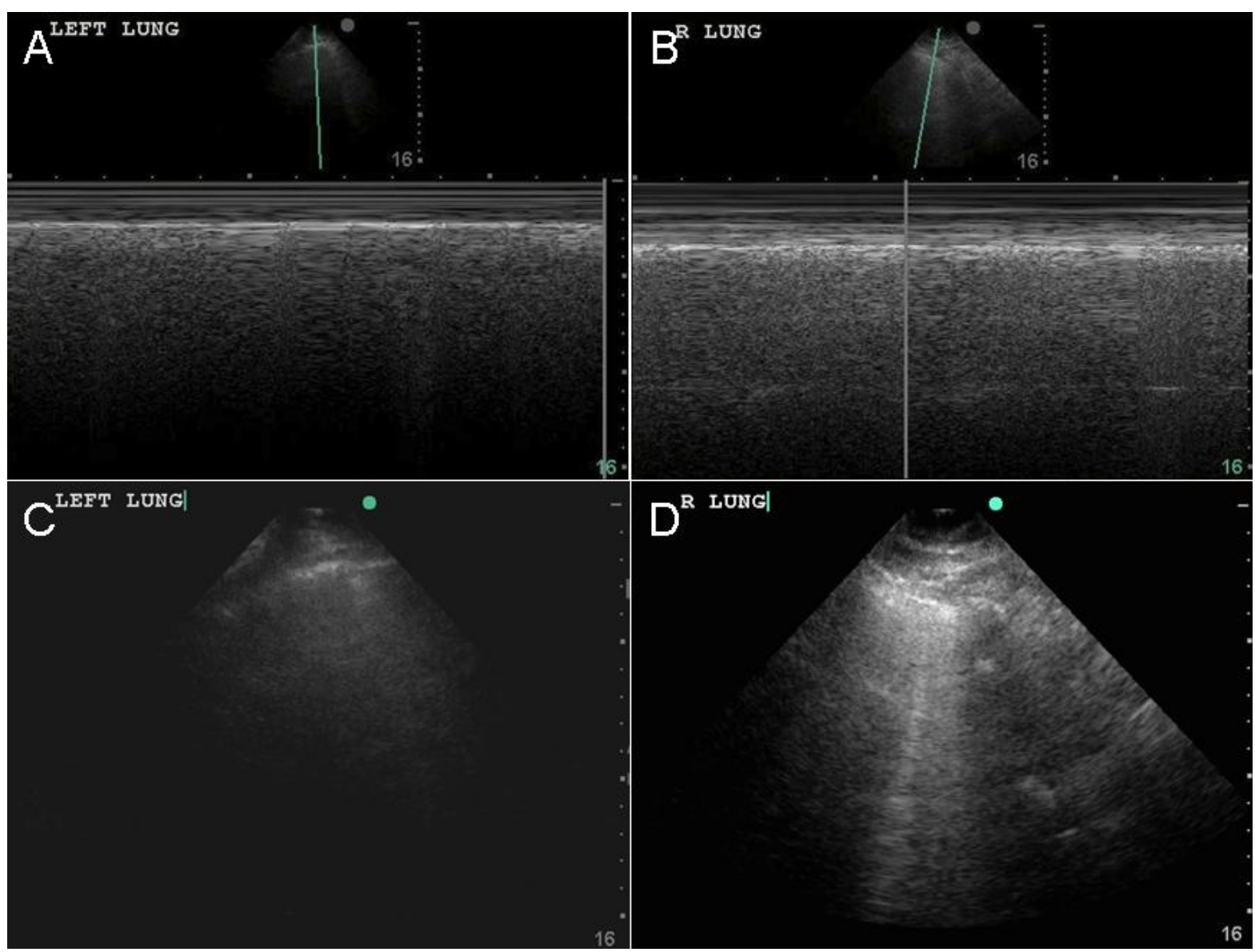

Figure 3. M mode of left lung (panel A) and the right lung (panel B). Ultrasound of left lung (panel C) and right lung (panel D). 
Which of the following are true?

1. There is a right-sided pneumothorax

2. There is bilateral interstitial edema

3. There is re-expansion pulmonary edema

4. 1 and 3

5. All of the above 


\section{Correct! \\ 3. Re-expansion pulmonary edema}

The M-modes in panels A and B show a normal "seashore" sign and not the "barcode" sign seen with a pneumothorax. Furthermore, there is lung sliding on both sides suggesting that a large pneumothorax is unlikely. The ultrasound shows "b" lines in the right lung in panel $D$. These are vertical lines radiating to the edge of the image. "b"

lines have been associated with interstitial edema. There are either no or few "b" lines in panel C. Given the clinical situation, the best explanation is unilateral pulmonary edema associated with re-expansion pulmonary edema. Although re-expansion pulmonary edema is more likely to occur with removal of pleural fluid of $>1500 \mathrm{ml}$, it can occur with removal of smaller volumes.

Which of the following is the treatment of choice for re-expansion pulmonary edema?

1. Intubation with mechanical ventilation

2. Diuresis

3. Supportive treatment

4. 1 and 3

5. All of the above 


\section{Correct! \\ 3. Supportive treatment}

Treatment of re-expansion pulmonary edema is supportive. Placing the patient with the affected side up is recommended which, in unilateral cases, contributes to reducing the pulmonary shunt and improving oxygenation. Noninvasive ventilation should be considered.

Our patient was treated with BiPAP and oxygen and gradually improved.

Prescriber Pearls (physicians/PAs/NPs) (Theodore Loftsgard R.N., C.N.P.)

1. Acute Hypoxic Respiratory Distress, pneumonia vs edema

a. History:

i. Co-mordities

ii. Recent pneumonia or exposure

iii. Recent procedures

b. Exam:

i. Auscultation: unequal, crackles, work of breathing

ii. Extremities: temperature, cap refill, color

iii. Bedside ultrasound: IVC, B lines, A lines, M mode, Effusions.

iv. Chest x-ray

v. Consider ABG

c. Differentials

i. Re-expansion pulmonary edema

1. Exam: Auscultation, Bedside Ultrasound, Chest x-ray

2. Treatment: CPAP/ BiPAP, Lasix, positioning

ii. Hospital Acquired Pneumonia

1. Cultures

2. Pulmonary hygiene

3. Antibiotics

iii. Pneumothorax related to procedure

1. Monitor close with increased oxygen

2. Chest tube

3. Avoid Positive Pressure Ventilation

iv. Thalidomide reaction

1. Monitor closely

2. Consider holding medication

2. Atrial fibrillation with rapid ventricular response

a. History:

i. Co-mordities

ii. Recent pneumonia or exposure

iii. Recent procedures

b. Exam:

i. Auscultation: Murmur, gallop

ii. Extremities: temperature, cap refill, color

iii. Bedside ultrasound: IVC, LV size and function, RV size function 
iv. EKG, verify rhythm

v. Electrolytes

c. Differentials

i. Fluid Overload

ii. Low Potassium, Magnesium, phosphorus.

iii. Hypothyroidism

3. Assessment of volume status in ICU

a. Data gathering: prior intake and output trends: Diarrhea, vomiting, malnutrition, changes in urine output, sweat production, prior edema. Weakness onset, polyuria, polydipsia

b. Exam:

i. Skin and mucous membranes: moist or dry. Skin tenting?

ii. Vitals: Postural hypotension? Dizziness?

iii. Laboratory: Urine output currently? Elevation in Creatinine and BUN. Urine osmolality, FENa, abnormal electrolytes.

c. Advanced measurements/ responsiveness $(1,2)$

i. $\quad$ CVP (if central access obtained): This can be used as a guide on volume status. CVP does not adequately predict whether or not an intravenous fluid challenge will increase stroke volume and cardiac index.

ii. CCS bedside ultrasound: Among spontaneously breathing patients, the IVC diameter is a robust estimate of central venous pressure

iii. $\quad$ Passive Leg Raise: PLR induces larger increase in cardiac preload than and may be preferred for predicting fluid responsiveness.

- Nursing Pearls (Dacia Evans R.N.)

- Admission reason: 1900 Tachypnea, dyspneic, labored breathing; maintaining oxygen saturations, Afib

- Respiratory Assessment

o Recent thoracentesis with $1 \mathrm{~L}$ off; concern for pulmonary edema

- Placed on CPAP 7 with 40\%, appeared labored with use of accessory muscles

- Comfortable and resting on CPAP

- Saturations good 98-99\%

- Quality/depth/rate of respirations adequate

- 0100- placed on BiPAP for concern for $\mathrm{CO} 2$ retention due to change in neurologic status

o 0600- placed on 2Lncann

- Cardiac assessment

o Sinus tachycardia in the 130's with frequent PACS, BP stable in the 110130 range

- 2200 after oral dose of Lopressor given, HR in 90's

- Magnesium and Calcium replete 
- IV Lopressor given for HR goal of $<100$ (20 mg given)

o No urine output overnight, initially incontinent on admission to ICU

- Concerned for dehydration, voided at 0600 for 1000cc's

o Hemoglobin on ABG at 0100-6.2 (2 RBCS ordered and administered)

- Neurological assessment

o Baseline assessment: patient A \&0x3, conversing, RASS -1

- 2130 patient awoke, swallowed medications and returned to CPAP

o Unresponsiveness at 2300; responsive to sternal rub and painful stimuli only, RASS -4

- Q1 hr neurological assessments from 2300 to 0400 without change; pupils remained equal and reactive, only responsive to painful stimuli

- Patient became more responsive at $A \& 0 \times 3$ at $4 a m$

o Concern for $\mathrm{CO} 2$ retention?

- Service notified, oxygenation ok

- ABG: showed no CO2 retention

- CPAP changed to BiPAP due to concern for ventilation

o Concern for neurological process?

- Pupils equal and reactive

- Medications administered at 2130; thalidomide (oral chemotherapy), metoprolol 50mg PO

o Prior to admission to ICU 18000.5 mg PO Lorazepam

Respiratory Therapy Pearls (Adam Frost R.R.T., C.R.T.)

Underlying respiratory disease (asthma): usually treat with albuterol, but monitor heart rate (tachycardia). Our patient's heart rates were from 110-170 so we should treat with ipratropium which does not affect heart rate as much as epinephrine.

When looking at that chest CT the patient was found to have a pleural effusion and atelectasis. The ICU service tapped the effusion which should in return help with patient work of breathing, lung expansion, and the atelectasis. Respiratory can assist with improving the atelectasis by performing bronchial hygiene with the patient by working with incentive spirometry (I.S.) to help the patient reinflate the alveoli in the lung to eliminate the atelectasis. If the patient is not strong enough to use the I.S., the respiratory therapist could try EZPAP, which utilizes a fluidic process to augment spontaneous breathing to provide higher inspiratory flow and larger tidal volumes than on unsupported ventilation. The patient also exhales against resistance, creating PEEP (Positive End Expiratory Pressure) to stent the airways open and promote lung recruitment. The flow to the device should be initiated at a lower flow and increased slowly as the patient tolerates. Patient should be assessed pre and post therapy and monitored for any signs of distress as therapy will temporarily increase patient's work of breathing. Patient should be allowed to take rest periods during the treatment. 
When the patient arrived in the ICU she was having increased work of breathing, to help the patient out we started her on 30\% FiO2 and CPAP 7. CPAP is Continuous Positive Airway Pressure is a spontaneous breath mode during which there are no mechanically delivered inspirations. Advantages of CPAP include significantly lower peak airway pressures for a given tidal volume, spontaneous breathing throughout the ventilatory cycle for patient comfort, due to the presence of spontaneous breathing, hemodynamic compromise such as can occur with PEEP during mechanical ventilation rarely occurs, decreased sedation, and elimination of neuromuscular blockade use. Contraindications for CPAP include apnea or hypoventilation, increasing sedation or administration of a paralytic, and impaired ventilatory drive.

During the patients stay in the ICU while she was using CPAP for support, the patient had a decrease in mental status and was having periods of apnea due to some side effects of the medicines she had received earlier before coming to the ICU. To help the patient out we placed her on BIPAP, is delivering IPAP (Inspiratory Positive Airway Pressure) and EPAP (Expiratory Positive Airway Pressure). Also referred to as bi-level PAP therapy. BIPAP therapy is used to treat sleep related disorders including severe COPD, OSA and nocturnal hypoventilation. We placed the patient on BIPAP with settings, 30\% FiO2 S/T 4 (spontaneous/timed: this mode lets you breath at your own rate with giving you the support the therapist sets or if the patient does not take a breath in the allotted time the machine will give the patient a breath,) with an IPAP of 14 and EPAP of 7 giving the patient a pressure support of 7 . Contraindications for BIPAP are patents unable to protect airway or adequately clear secretions, with a high risk for aspiration, acute sinusitis, severe respiratory therapy patients without a spontaneous respiratory drive, pneumothorax, recent gastric, laryngeal, or esophageal surgery, significant facial fractures, nausea and vomiting, and cardiac ischemia or acute myocardial infarction. This patient had none of these. The patient was oxygenating well just not ventilating that is why we made the Switch to BIPAP from CPAP.

\section{Pharmacy Pearls (Karen C. Kolbet, Pharm.D., R.Ph.)}

Thalomid $®$ (thalidomide): known in the $1950-60$ s as a sedative and hypnotic.

Mechanism of action: In the treatment of multiple myeloma patients, thalidomide is thought to work through several mechanisms including induction of apoptosis, inhibition of vascular endothelial growth factor, modulation of immune surveillance, and decreased adherence of myeloma cells to bone marrow stromal cells.

Indications for use: It is FDA approved for use in multiple myeloma as monotherapy and in several combination regimens.

Black Boxed Warning: Thalidomide does contain a black boxed warning for an increased risk of venous thromboembolic events when used in patients with multiple myeloma. This risk is increased when thalidomide is used in combination with other chemotherapeutic agents. 
Incidence of pulmonary/respiratory adverse effects: Prescribing information: Based on results from a Celgene-sponsored controlled clinical trial (4):

i. Respiratory system adverse effects were reported in 3 (12.5\%) of patients:

-Pharyngitis: 1 (4.2\%)

-Rhinitis: 1 (4.2\%)

-Sinusitis: 1 (4.2\%)

ii. Treatment-emergent adverse effects occurred in $>l=10 \%$ of all patients -Grade 3/4 pulmonary adverse events occurred in $52(51 \%)$ of patients

a) Dyspnea: $43(42.2 \%)$

b) Cough: $15(14.7 \%)$

\section{DTPACE:}

i. Pulmonary and renal toxicities and metabolic abnormalities were brief, lasting less than 1 week after completion of 4-day chemotherapy

ii. After the first cycle of DTPACE, 6 patients (3\%) died as a result of treatmentrelated causes:

-Respiratory failure caused by bacterial pneumonia $(n=1)$

iii. After the second cycle of DTPACE 3 additional patients died:

-Sepsis $(n=2)$

-Cytomegalovirus pneumonia $(n=1)$

iv. Overall incidence of pulmonary toxicity of chemotherapy regimen:

-Dyspnea 21 (5\%)

-Pulmonary infiltrates 7 (2\%)

-Pulmonary edema 5 (1\%)

Case Report: Thalidomide-induced pneumonitis:

A 71 year old man with a history of stage IIIB symptomatic multiple myeloma was admitted with fever and dyspnea. He was on oral thalidomide $200 \mathrm{mg} /$ day in combination with melphalan, cyclophosphamide, and dexamethasone. After 12 days of therapy with thalidomide, he became febrile and crackles were present on pulmonary exam. After a negative infectious work-up, thalidomide and antimicrobials were discontinued. His symptoms resolved in approximately 1 week. Upon discharge thalidomide was resumed in which case his dyspnea and fever recurred $\sim 8$ days later (5).

Case Report: Interstitial pneumonitis as an adverse effect of thalidomide: A 67 year old man with history of multiple myeloma on thalidomide (approximate duration completed 2 months) in combination with dexamethasone was admitted with progressive dyspnea. CT scan showed diffuse ground glass opacities in both lungs however infectious etiology was ruled out. Thalidomide was discontinued and ground glass opacities and dyspnea resolved (3).

Case Report: Thalidomide-induced reversible interstitial pneumonitis in a patient with recurrent ovarian cancer:

A 58 year old woman with a history of recurrent ovarian cancer on thalidomide $200 \mathrm{mg} /$ day (approximate duration completed 5 weeks) in combination with weekly topotecan developed shortness of breath and dyspnea on exertion with a non- 
productive cough. Work-up for infectious etiology versus $\mathrm{PE}$ remained negative despite concerning CT for opportunistic infection. Thalidomide was subsequently held in which her shortness of breath completely resolved without intervention. Repeat CT showed resolution of ground-glass alveolar opacities and micro nodules present on previous image.

Of note, some key contributing factors to note about our patient include a past medical history of asthma, eczema, and h/o pneumonia as well as the concurrent use of narcotics which can decrease respiratory drive putting the patient at an increased risk for respiratory infections. Our patient was also neutropenic which made them more susceptible to not only routine infections, but opportunistic organisms as well.

\section{References}

1. Prekker ME, Scott NL, Hart D, Sprenkle MD, Leatherman JW. Point-of-care ultrasound to estimate central venous pressure: a comparison of three techniques. Crit Care Med. 2013;41(3):833-41. [CrossRef] [PubMed]

2. Jabot J, Teboul JL, Richard C, Monnet X. Passive leg raising for predicting fluid responsiveness: importance of the postural change. Intensive Care Med. 2009;35(1):85-90. [CrossRef] [PubMed]

3. Buttin BM, Moore MJ. Thalidomide-induced reversible interstitial pneumonitis in a patient with recurrent ovarian cancer. Gynecol Oncol. 2008;111(3):546-8. [CrossRef] [PubMed]

4. Lee CK, Barlogie B, Munshi N, Zangari M, Fassas A, Jacobson J, van Rhee F, Cottler-Fox M, Muwalla F, Tricot G. DTPACE: an effective, novel combination chemotherapy with thalidomide for previously treated patients with myeloma. J Clin Oncol. 2003;21(14):2732-9. [CrossRef] [PubMed]

5. Scholte JB, Potjewijd J, Voogt PJ, Custers FL, Jie KS. Interstitial pneumonitis as an adverse effect of thalidomide. Ned Tijdschr Geneeskd. 2009;153:A452. [PubMed]

6. Thalomid (thalidomide): Highlights of Prescribing Information. (2014, June 1). Retrieved February 6, 2015, from http://www.thalomid.com/pdf/thalomid pi.pdf

7. Wyplosz B, Lerolle U, Israël-Biet D, Dougados J, Lillo-Le Louët A, Ghez D, Pouchot J. Thalidomide-induced pneumonitis. Eur J Intern Med. 2008;19(7):e57-8. [CrossRef] [PubMed] 